\title{
Depletion of cutaneous nerves and neuropeptides in diabetes mellitus: an immunocytochemical study
}

\author{
D. M. Levy ${ }^{1}$, S.S. Karanth ${ }^{2}$, D. R. Springall ${ }^{2}$ and J. M. Polak ${ }^{2}$ \\ ${ }^{1}$ Department of Diabetes and Endocrinology, Central Middlesex Hospital, and \\ ${ }^{2}$ Department of Histochemistry, Royal Postgraduate Medical School, London, UK
}

\begin{abstract}
Summary. Immunocytochemistry for the general neuronal marker protein gene product 9.5 and four neuropeptides (calcitonin gene-related peptide, substance $P$, vasoactive intestinal polypeptide and neuropeptide $Y$ ) was performed on 20 skin biopsy specimens from 19 diabetic patients, age range 20-75 years, 17 Type 2 (non-insulin-dependent) and 3 Type 1 (insulin-dependent). Fifteen specimens were from the lower limb, 3 from the upper limb und 2 from the abdominal wall. Seven subjects had lower limb neurophysiological tests. All but one specimen showed reduced protein gene product 9.5 and neuropeptide immunoreactivity. Reduced protein gene product 9.5 and neuropeptide immunoreactivity was found in specimens taken from the abdominal wall and hand as well as
\end{abstract}

those from the leg, and also in specimens from patients undergoing amputation for peripheral vascular disease. In general, the greater the number of abnormal neurophysiological tests, the greater the extent of neuronal abnormalities. Three patients with normal tests had abnormalities of dermal innervation. While these changes are also found in other axonal neuropathies, in the absence of other causes of peripheral nerve disease and of macrovascular disease, immunocytochemistry of skin biopsies may have a role in the assessment of diabetic neuropathy and its response to treatment.

Key words: Diabetic neuropathy, skin, immunocytochemistry, neuropeptides, neurophysiology.
While diabetic nerve damage is the commonest chronic complication of diabetes [1], its aetiology is still poorly understood. Its response to apparently rational therapies, for example aldose reductase inhibitors [2] and gangliosides [3] is inconsistent, and this may in part be due to the indirect, predominantly neurophysiological methods currently used in diagnosis and follow-up, which have high variability [4], and which reflect function in only one group of nerve fibres. Sural nerve fascicular biopsy has been useful in the study of the morphometry of diabetic nerve [5], but it is a specialised technique that currently has limited applicability. A histological method is required that more directly reflects minor and subclinical deficits of nerve function, especially in peripheral sensory and autonomic fibres.

Immunocytochemical techniques, now in routine use in many diagnostic pathology laboratories, have already been used to map the distribution of neuropeptides in the peripheral sensory and autonomic fibres of the skin [6]. Such studies have shown that substance $P$ (SP) and calcitonin gene-related peptide (CGRP) are localised in sensory $\mathrm{C}$ fibres [7], and are believed to play an important role in neurogenic inflammation and the microvascular leakage elicited by axon reflexes $[8,9]$.
Neuropeptides associated with peripheral autonomic innervation include vasoactive intestinal polypeptide (VIP) and neuropeptide Y (NPY). VIP is a potent vasodilator, and VIP-immunoreactivity is low in the corpora cavernosa of diabetic and non-diabetic impotent men [10,11]. It is also co-localised with acetylcholine in sudomotor nerves [12], and both substances probably play a role in sweat-gland function, which is frequently abnormal in diabetes $[13,14]$. CGRP has recently been found co-localised with VIP in nerves around sweat glands [15]. NPY is also found in peripheral autonomic nerves, where it may play a vasoconstrictor function [16, 17]. In addition to these specific neuropeptides, several general neuronal markers have been described. They are associated with all types of mammalian nerves and include neuron-specific enolase [18], neurofilament triplet proteins [19] and protein gene product (PGP) 9.5 [20, 21].

The widespread peripheral neurological abnormalities of diabetes might therefore be reflected in abnormalities of skin neuropeptides, and any changes may occur at an early stage, as has been suggested for leprous neuropathy [22]. We have therefore investigated skin biopsies from a variety of sites in normal (non- 
Table 1. Clinical characteristics of patients, neurophysiological and immunohistochemical findings

\begin{tabular}{lllllllllllllll}
\hline $\begin{array}{l}\text { Speci- } \\
\text { men }\end{array}$ \\
no.
\end{tabular}

${ }^{a} \mathrm{PVD}=$ peripheral vascular disease (absent foot pulses); ${ }^{\mathrm{h}}$ Abnormal common peroneal motor conduction velocity ( $>2 \mathrm{SD}$ below age-related normal valuc); 'Sensortek threshold $>1.1^{\circ} \mathrm{C}$; ${ }^{\mathrm{d}}$ Any two of the following: (a) heart rate variation with deep breathing $<11$ beats per minute; (b) Valsalva ratio $<1.11$; (c) $30: 15$ ratio $<1.04$; ${ }^{\mathrm{e}} \mathrm{OH}=$ oral hypoglycaemic agent(s); ${ }^{\mathrm{f}}$ Normal peroneal conduction velocity but absent medial plantar sensory action potential; ' No sweat glands seen in this specimen

Semi-quantitative scalc of immunoreactive fibre density: absent $(-)$, few $(+)$, moderate $(++)$, many $(+++)$, abundant $(++++)$ PGP9.5= protein gene product 9.5 , VIP = vasoactive intestinal polypeptide, $C G R P=$ calcitonin gene-related peptide, $S P=$ substance $P$, VPY $=$ neuropeptide $Y$

diabetic) and diabetic subjects, using immunocytochemical methods. Some subjects have also had neurophysiological, sensory threshold and vagal cardiovascular autonomic tests carried out.

\section{Meterials and methods}

Skin biopsies were taken from 19 consecutive diabetic patients undergoing clective general surgical and orthopedic operations. Their clinical details are given in Table 1 . Their ages ranged from 20-75 years and the time since diagnosis from 0.534 years. Three were Type 1 (insulin-dependent), the remainder Type 2 (non-insulin-dependent). None were ketotic or hyperosmolar at the time of surgery. One patient had known chronic alcoholic liver disease; no others were known to have untreated endocrine or other disease that might affect peripheral nerve function. Patients were considered to have symptomatic neuropathy if they had any spontaneous distal paraesthesia, numbness, burning or contact discomfort. Six patients had peripheral vascular disease, as assessed by absent foot pulses; five of them were undergoing lower limb amputation and two had characteristic symptoms of long-standing painful diabetic neuropathy. Control specimens were age- and site-matched and taken from patients undergoing orthopedic or general surgical procedures. None had neurological disease, alcoholism, diabetes or other endocrine or systemic diseases known to affect peripheral nerve or vascular function.

Full-thickness skin specimens approximately $2 \mathrm{~cm} \times 0.3-0.5 \mathrm{~cm}$ were taken from the margin of the surgical incision at the time of operation, care being taken not to crush the specimens. One patient had two biopsies taken at the same time, from the abdomen and the calf. Amputation specimens were taken from the proximal viable margin, and all amputations subsequently healed uneventfully. Specimens were immediately fixed (1) for approximately $2 \mathrm{~h}$ in $0.4 \%$ solution of p-benzoquinone in phosphate-buffered saline (PBS: $0.01 \mathrm{~mol} / 1$ phos- phate buffer, $\mathrm{pH} 7.2$, containing $0.15 \mathrm{~mol} / 1$ sodium chloride), or (2) overnight in Zamboni's solution; both were then transferred to PBS containing 7\% sucrose and $0.01 \%$ sodium azide.

The results of two or more neurological function tests were available for seven patients. Neurophysiological examinations were carried out according to previously published methods [23]. Thermal thresholds were measured with the Sensortek apparatus [24] and cardiac vagal autonomic function by a computerised method. Results were considered abnormal if they fell outside $95 \%$ confidence limits for age-related normal subjects, or, in the case of autonomic tests, 2 or 3 were clearly abnormal by Ewing and Clarke's criteria [25].

The study was approved by the Ethics Committee of Brent Health Authority.

\section{Immunocytochemistry}

Cryostat sections $(6 \mu \mathrm{m})$ were taken up on poly-L-lysine coated glass slides, allowed to dry for $2 \mathrm{~h}$ at room temperature and then placed in PBS containing $0.2 \%$ Triton X-100 for $30 \mathrm{~min}$. A modified indirect immunofluorescence staining method [22] was used for immunostaining, with antisera to general neuronal marker (PGP 9.5) and to the neuropeptides VIP, CGRP, SP and NPY (Table 2). Three non-serial sections were used for each antiserum. Sections were incubated in appropriately diluted primary antiserum for $16 \mathrm{~h}$ at $4^{\circ} \mathrm{C}$, then washed thoroughly for $10 \mathrm{~min}$ in three fresh changes of PBS and reincubated in the primary antiserum for $3 \mathrm{~h}$ at room temperature. After threc further 5-min washes in PBS, goat anti-rabbit FITC conjugate $(1: 200$, Miles Laboratories, Stoke Poges, UK) was applied to the sections for $60 \mathrm{~min}$. Slides were washed again for $5 \mathrm{~min}$ in three fresh changes of PBS, mounted in PBS glycerol (1:9 volume/volume) and viewed using a fluorescence microscope.

Numbers of immunoreactive fibres in each specimen were assessed visually and the results graded from - (absent) to ++++ (abundant) (see Table 1). 


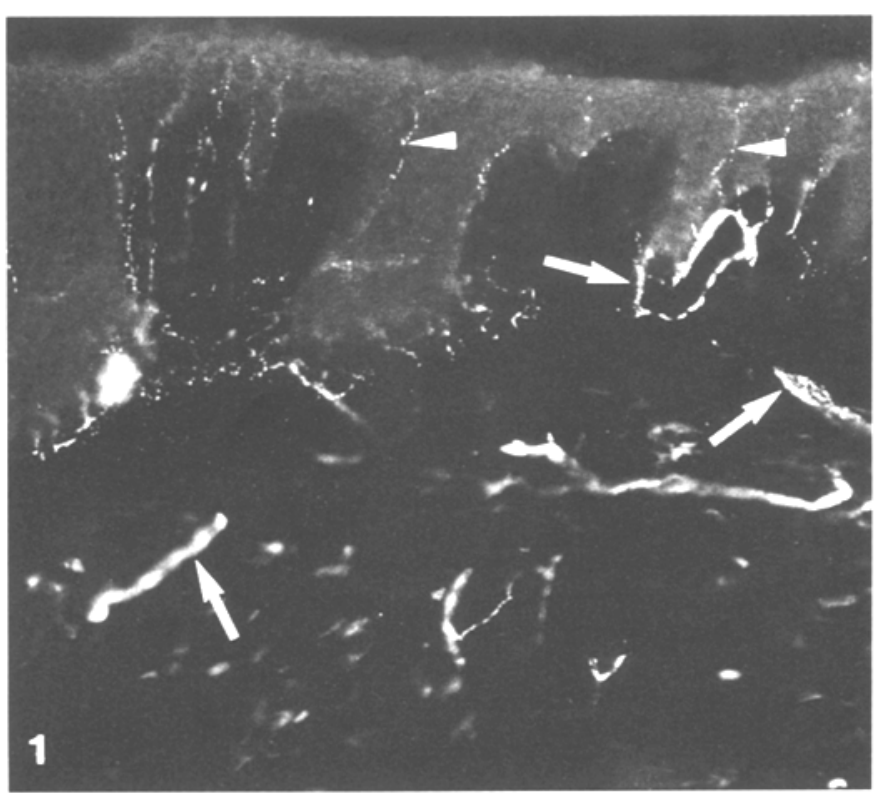

Fig. 1 and 2. Skin of calf showing PGP-immunoreactive fibres in epidermis (arrow head) and dermis (arrow) of non-diabetic (Fig. 1) and diabetic (Fig. 2) skin. Note the reduction in density and number of immunoreactive fibres, particularly in the epidermis of diabetic skin. Zamboni-fixed tissues, cryostat sections $(10 \mathrm{um})$, indirect immunofluorescence with anti-PGP 9.5 serum

All sections were assessed by the same observer (SSK) and $10 \%$ of them checked by another; both were unaware of the patients' diabetic or neuropathic status. Results were compared with those for age- and site-matched control subjects.

\section{Results}

\section{Control specimens}

The distribution of nerve fibres immunoreactive for PGP 9.5 and neuropeptides was identical to that previously reported [18]. All control specimens were graded ++++ for PGP $9.5,+++$ for CGRP and VIP, and ++ for SP and NPY (Table 1). PGP-like immunoreactivity was observed in free nerve endings in epidermis and dermis, around the hair follicles and sweat glands. Nerve bundles related to blood vessels and arrector pili muscles showed similar immunoreactivity. CGRP- and substance P-immunoreactive fibres usually appeared as beaded fibres in the dermis, though a few extended into the epidermis. Substance P-immunoreactive nerves, less common than CGRP-immunoractive nerves, were found near to sweat glands and hair follicles. NPY- and VIP-immunoreactivity was found near sweat glands and blood vessels, though NPY-immunoreactivity was lower both in frequency and intensity. Specimens of skin from normal subjects immunostained for PGP 9.5, VIP and CGRP are shown in Figures 1, 3 and 5, respectively.

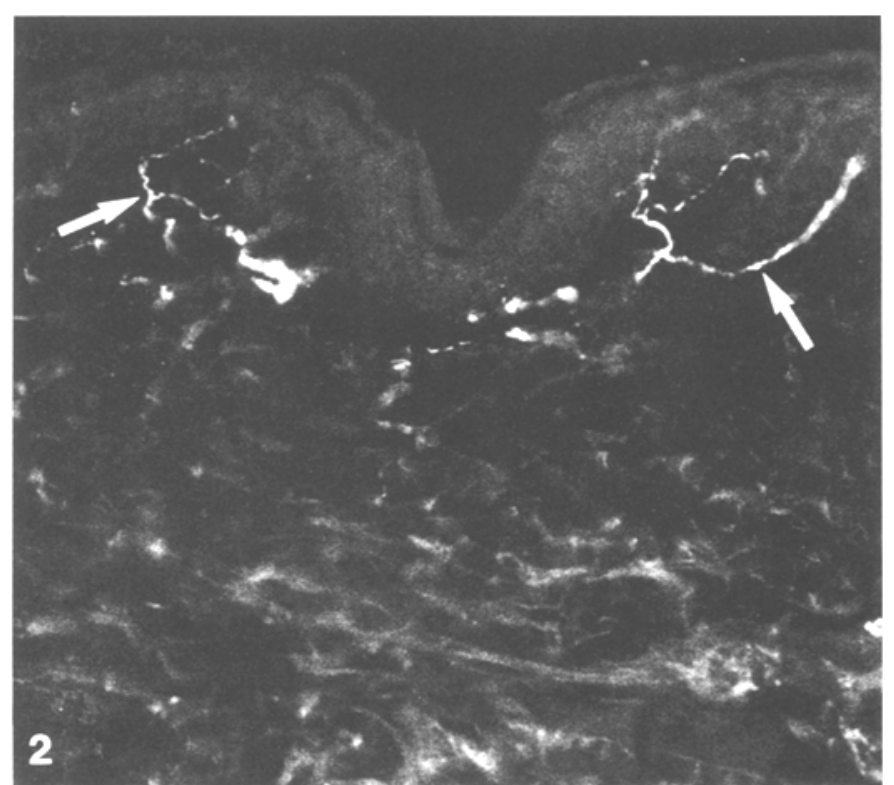

\section{Diabetic specimens}

General neuronal marker PGP 9.5. A summary of the immunocytochemical findings is presented in Table 1. One specimen (no.7, from the medial side of the wrist) had normal PGP 9.5 and neuropeptide immunoreactivity. The remaining 19 diabetic specimens had lower numbers of PGP 9.5-immunoreactive fibres than control specimens, and within each fibre immunoreactivity was less intense. However, only those specimens (nos. 4, 14 and 15) from patients with severe peripheral vascular disease were devoid of PGP9.5-immunoreactivity. Figure 2 shows PGP 9.5-immunoreactivity in a skin specimen from a diabetic patient.

\section{Neuropeptides}

Neuropeptides present in sensory nerves (SP. CGRP). All but one diabetic specimen (no.7) had diminished levels of $\mathrm{SP}$ and CGRP. The widespread diminution of $\mathrm{SP}$ was particularly striking in specimen 9 , from a 31-year-oldType 2 diabetic, diagnosed 6 months before operation, and in specimen 10 , from a 20 -year-old Type 1 diabetic patient, duration 12 years. There was no SP-immunoreactivity in specimens 2 and 3 , from the same patient; he had elevated thermal thresholds and abnormal cardiac autonomic function, but results of his neurophysiological tests were normal. All specimens, apart from no.7, also showed decreased CGRP-immunoreactivity, though the pattern of decreased SP-immunoreactivity did not uniformly reflect that of CGRP; for example, the two specimens taken from the same patient (nos. 2 and 3) demonstrated more CGRP-immunoreactivity in the abdominal skin specimen than in the specimen from the calf. Figure 6 shows a specimen from a diabetic patient, immunostained for CGRP. 

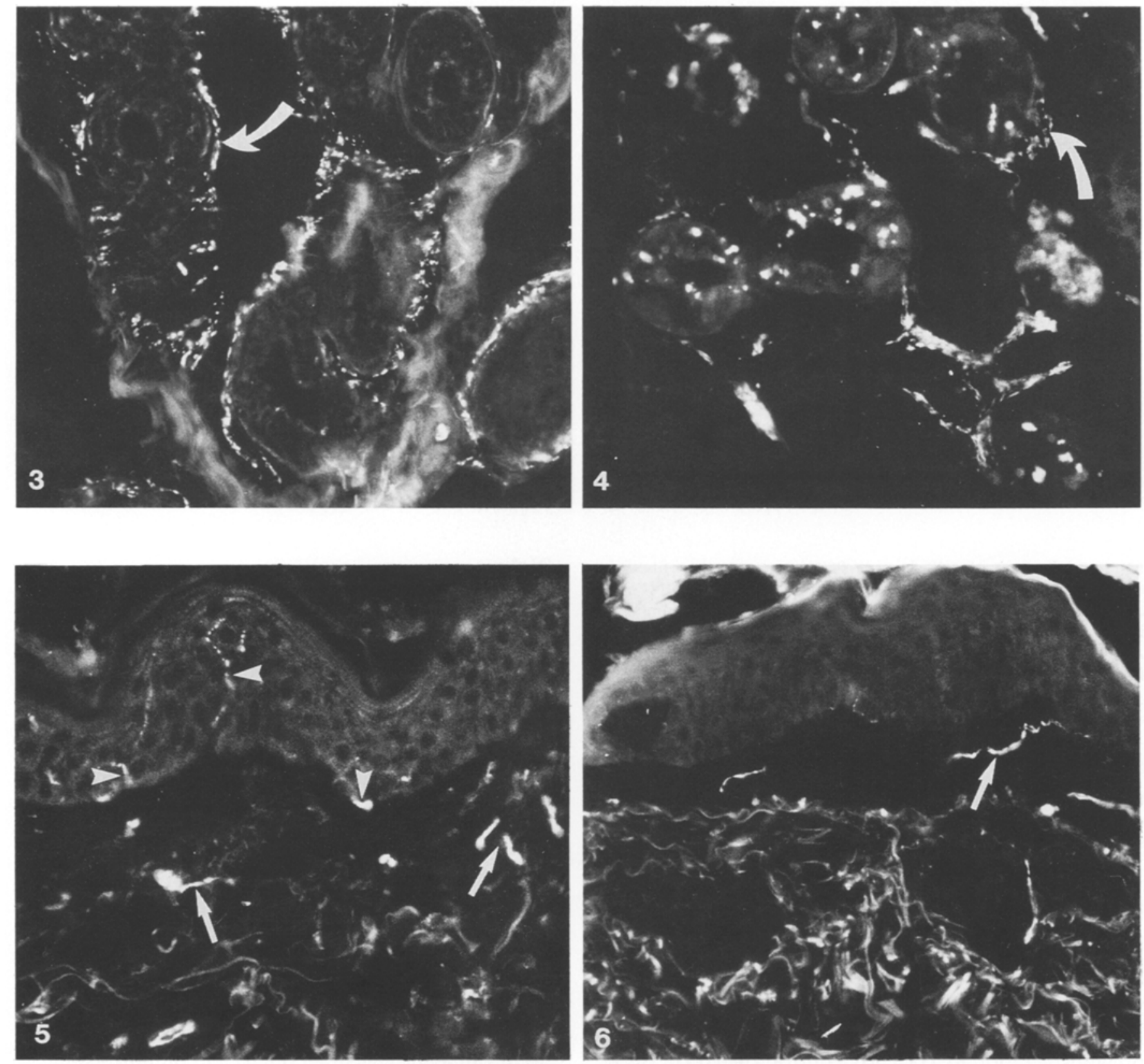

Fig. 3 and 4. Specimens of calf skin immunostained with VIP antiserum, showing immunoreactive fibres around sweat glands in nondiabetic (Fig.3) and diabetic (Fig.4) skin. The number of fibres is greatly reduced in the diabetic specimen

Fig.5 and 6. Skin of calf showing CGRP-immunoreactive fibres in epidermis (arrow head) and dermis (arrow) of non diabetic (Fig.5) and diabetic (Fig.6) skin. Note the difference in the numbers of fibres

Neuropeptides present in autonomic nerves (VIP, NPY). Apart from specimen no. 7 all diabetic specimens had diminished VIP- and NPY-immunoreactivity. There was a closer association between VIP and NPY levels than between SP and CGRP. They were only entirely absent in amputation specimens from patients with

known peripheral vascular disease. In Figure 4 a skin specimen from a diabetic patient shows reduced numbers of VIP-immunoreactive fibres around sweat glands.

\section{Neurophysiological tests}

In the seven patients with neurophysiological tests, there was a broad association between the number of abnormal tests and the immunohistochemical abnormalities (Table 1). However, three subjects who had normal thermal thresholds and autonomic function (nos. 5, 6 and 8) had widely differing neuropeptide immunoreactivities, though the skin sites were also differ- 
Table 2. Antisera used in immunocytochemistry

\begin{tabular}{llll}
\hline $\begin{array}{l}\text { Rabbit } \\
\text { antiserum } \\
\text { to }\end{array}$ & Source & Dilution & $\begin{array}{l}\text { Absorp- } \\
\text { tion }\end{array}$ \\
\hline PGP 9.5 & Ultraclone, Cambridge, UK & $1: 1000$ & \\
CGRP & Hammersmith Hospital, London, UK & $1: 200$ & 0.1 \\
SP & Hammersmith Hospital, I ondon, LK & $1: 100$ & 1.0 \\
NPY & Hammersmith Hospital, London, LK & $1: 400$ & 1.0 \\
VIP & Hammersmith Hospital, London, UK & $1: 200$ & 1.0 \\
\hline
\end{tabular}

a Concentration of synthetic antigen required to abolish immunostaining ( $\mathrm{nmol} / \mathrm{ml}$ of diluted antiserum)

PGP 9.5 = protein gene product 9.5; $C$ GRP $=$ calcitonin gene-related peptide: $S P=$ substance $P ; N P Y=$ neuropeptide $Y ; V I P=$ vasoactive intestinal polypeptide

ent. One of these specimens (no.8) was taken from a patient who had normal routine electrophysiological tests (common peroneal motor conduction velocity, sural sensory action potential amplitude and conduction velocity) but whose medial plantar response (the most distal sensory electrophysiological test currently available) was absent [24]. Both specimens from patients with painful diabetic neuropathy (nos. 1 and 4 ) had virtually absent immunoreactivity, though it should be noted that they both had long-standing symptoms, and also peripheral vascular disease.

\section{Discussion}

The current study demonstrates that nerves of the skin are frequently diminished in diabetes, as shown by immunostaining of the general neuronal marker PGP 9.5 and neuropeptides. Abnormalities were seen in all but one of twenty specimens.

There have been two previous studies of dermal nerves in diabetes. Faerman et al. [26], using conventional argyrophilic techniques and light microscopy, demonstrated significant abnormalities of autonomic nerve structure. Johnson and Doll [27] found non-vascular perineurial cell basement membrane thickening. In Faerman's study there was an association between anhidrosis, as assessed by an abnormal sweat test, and histopathological abnormalities of small fibres in the skin, particularly those near to sweat glands. It is likely that these changes are relatively late, as clinical abnormalitics of sudomotor function are usually found in association with clinically evident polyneuropathy; they are not specific to diabetes, however, since sympathectomised non-diabetic patients showed the same histological abnormalities.

In this study, we have used immunocytochemical staining both for the general neuronal marker PGP 9.5 and four specific neuropeptides. PGP $9.5[20,21]$ probably gives an indication of the presence and structural integrity of nerve fibres. Neuropeptide immunoreactivity is more difficult to interpret, as it is a reflection of the amount of stored neuropeptide, and this is influenced by synthesis, transport, release and degradation. Assuming that nerves are present, it reflects the overall functional state of the nerve. The abnormalities seen in this study therefore represent both functional and structural alterations in cutaneous nerves.

Whatever the known duration of diabetes, density and distribution of immunoreactive nerves in all but one specimen was abnormal compared to control specimens; the specimen with normal immunoreactivity was taken from the upper limb, where clinical and neurophysiological evidence of neuropathy occurs late. Conversely, the known duration of diabetes in patient 9 , with abnormal immunocytochemical findings in the foot, was less than 1 year.

The number of patients with neurophysiological tests was small, and further studies are required to define more precisely the relationship between immunocytochemical abnormalities and functional neurological assessments. However, the findings in the proximal (abdominal) and distal (calf) specimens taken simultaneously from the same patient with known neurophysiological abnormalities suggest there may be a proximal-distal gradient of PGP 9.5 and neuropeptide immunoreactivity similar to that shown for electrophysiology [28].

The pattern of immunocytochemical depletion was broadly similar, with approximately $50 \%$ of specimens being devoid of immunoreactivity to each of the four neuropeptides. Within each specimen there was a similar degree of depletion. SP-immunoreactivity was most consistently reduced or absent, even in the two recently diagnosed subjects. Abnormalities of SP in diabetes are interesting, in view of the lower pain thresholds found in diabetic subjects [29] and their predisposition to abnormal pain perception. Patients with clinical and neurophysiological evidence of diabetic neuropathy also have decreased flare responses to intradermal SP [30], suggesting receptor changes that may follow from a decrease in SP-immunoreactive nerves. Axon reflex flare responses are also reduced in patients with peripheral vascular disease [31], and our finding of virtually absent PGP 9.5 and neuropeptides in specimens from these patients would be an expected corollary. While immunocytochemical abnormalities appear from this study to be exacerbated by large-vessel ischaemia, they are unlikely to be the sole cause of them, since abnormal results were found in specimens from areas usually unaffected by large vessel disease (eg anterior abdominal wall, dorsum of the hand). However, this study does not shed light on the role of microvascular disease in causing PGP 9.5 and neuropeptide depletion.

Neuropeptide depletion may be a common histopathological end-point in several neuropathies. Recent work [22] has shown that skin biopsies from patients with active lepromatous infection (an infective axonal ncuropathy) are uniformly depleted both of PGP 9.5 
and of neuropeptides, to a greater extent than in our diabetic subjects. This may be a manifestation of the focal nature of lepromatous neuropathy; a picture similar to that in diabetes may be found in other "metabolic" neuropathies of the axonal type. This needs to be confirmed, but is suggested by the severe immunocytochemical depletion found in specimen 13, from a patient with chronic alcoholic liver disease and intermittent hepatic failure, but who had only recently been diagnosed diabetic. One of the underlying abnormalities in peripheral neuropathies may be deranged axonal transport [32], and this would explain the broad associations between depletion of the various neuropeptides. Decreased axonal transport is an important early abnormality in diabetes, and one which is sensitive to changes in diabetic control, being reversed with insulin treatment in experimental models. No patients in the current study were metabolically decompensated though many had poor diabetic control. It will be important to study the effect of improved diabetic control and of therapy with drugs known to affect axonal transport (aldose reductase inhibitors and gangliosides [33]). Endoneurial hypoxia [34] which has been demonstrated both in experimental and human diabetes may be another factor contributing to the picture of reduced neuropeptide immunoreactivity.

Immunocytochemical abnormalities of skin biopsies of diabetic patients appear to be common, and broadly to reflect neuropathic status determined by standard neurophysiological tests. Further studies will be required to determine whether these abnormalities antedate neurophysiological abnormalities, and what their relationship is to symptoms and clinical findings. The direct histological assessment of peripheral nerves in diabetes is an important aim, and currently not routinely available; while important data on the pathophysiology of diabetic neuropathy and mode of action of therapeutic agents has resulted from sural nerve biopsies [35] it is not recommended as a routine procedure [36]. It cannot give information on the structure and function of the small peripheral fibres examined in this study, which are believed to play an important part in the symptoms of diabetic neuropathy [37]. Skin biopsy is safe, relatively atraumatic, and simple to perform, and the use of the techniques described in this study may give further insights into the mechanisms of peripheral nerve dysfunction in diabetes and its response to treatment at the histological level.

Acknowledgements. The co-operation of the orthopedic and general surgcons at Central Middlesex Hospital is gratefully acknowledged. Dr. Ralph Abraham gave much helpful advice. SSK held the Overseas Research Award from the Council of Vice-Chancellors and Principals of the United Kingdom. DMI, was a Fidia Rescarch Fellow. The peptide antisera used in this study were raised in collaboration with Professor S.R. Bloom, Department of Medicine, Royal Postgraduate Medical School, London.

\section{References}

1. Pirart J (1978) Diabetes mellitus and its degenerative complications: a prospective study of 4400 patients observed between 1947 and 1973. Diabetes Care 1: $168-188$

2. Zimmerman BR (1987) Current status of aldose reductase inhibitors. Diabetes Care 10: 123-125

3. Gorio A (1986) Ganglioside enhancement of neuronal differentiation, plasticity and repair. CRC Cr Rev Clin Neurobiol 2: 241-296

4. Fagius J, Wahren LK (1981) Variability of sensory threshold determination in clinical use. J Neurol Sci 51: 11-27

5. Behse F, Buchthal F, Carlsen F, Knappeis GG (1975) Unmyelinated fibres and Schwann cells of sural nerve in neuropathy. Brain 98: 492510

6. Bloom SR, Polak JM (1983) Regulatory peptides in the skin. Clin Exp Dermatol 8:3 318

7. Gazelius B, Edwall B, Olgart L, Lundberg JM, Hökfelt T, Fischer JA (1988) Vasodilatory effects and cocxistence of calcitonin generelated peptide (CGRP) and substance $P$ in sensory nerves of cat dental pulp. Acta Physiol Scand 130: 33-40

8. Foreman JC (1987) Peptides and neurogenic inflammation. $\mathrm{Br}$ Med Bull 43: 386400

9. Fuller RW, Conradson T-B, Dixon CMS, Crossman DC, Barnes PJ (1987) Sensory neuropeptide effects in human skin. Br J Pharmacol 92: 781-788

10. Gu J, Lazarides M, Pryor JP, Blank M , Polak JM, Morgan R, Marangos PJ, Bloom SR (1984) Decrease of vasoactive intestinal polypeptide (VIP) in the penises from impotent men. Lancet II: 315-317

11. Crowe R, Lincoln J, Blacklong PF, Pryor JP, Lumley JSP, Burnstock G (1983) Vasoactive intestinal polypeptide-like immunoreactive nerves in diabetic penis: a comparison between streptozotocin-treated rats and man. Diabetes 32: 1075-1077

12. Lundberg JM, Hökfelt T, Schultzberg M, Lvnäs-Wallenstein $K$, Köhler C, Said SI (1979) Occurrence of vasoactive intestinal polypeptide (VIP)-like immunoreactivity in certain cholinergic neurons of the cat: evidence from combined immunohistochemistry and acetylcholinesterase staining. Neuroscience 4: 1539-1559

13. Low PA, Caskey PE, Tuck RR, Fealey RD, Dyck PJ (1983) Quantitative sudomotor axon reflex test in normal and ncuropathic subjects. Ann Neurol 14: 573-580

14. Kennedy WR, Sakuta M, Sutherland D, Goctz FC (1984) Quantitation of the sweating deficiency in diabetes mellitus. Ann Neurol 15: $482-488$

15. Landis SC, Fredieu JR (1986) Coexistence of calcitonin gene-related peptide and vasoactive intestinal peptide in cholinergic sympathetic innervation of rat sweat glands. Brain Res 377: 177-181

16. Pernow J, Saria A, Lundberg JM (1986) Mechanisms underlying pre- and post-functional effects of neuropeptide $Y$ in sympathetic vascular control. Acta Physiol Scand 126: 239-249

17. Fdvinsson L, Fkman R, Jansen I, Ottosson A, Uddman R (1987) Peptide-containing nerve fibers in human cerebral arteries: immunocytochemistry, radioimmunoassay, and in vitro pharmacology. Ann Neurol 21: 431-437

18. Zomzeley-Neurath C, Walker W (1980) In: Bradshaw RA, Schneider DM (cds) Proteins of the nervous system, 2nd ed. Raven Press, Ncw York, pp 1-57

19. Hacker GW, Polak JM, Springall DR, Ballesta J, Cadieux A. Gu J, Trojanowski JQ, Dahl D, Marangos PJ (1985) Antibodies to neurofilament protein and other brain proteins reveal the innervation of peripheral organs. Histochemistry $82: 581-593$

20. Thompson RJ, Doran JF, Jackson P, Dhillon AP, Rode J (1983) PGP 9.5 a new marker for vertebrate neurons and neuroendocrine cells. Brain Res 278: 224228

21. Gulbenkian S, Wharton JM, Polak JM (1987) The visualisation of cardiovascular innervation in the guinea pig using an antiserum to protein gene product 9.5 (PGP9.5). J Autonom Nerv Syst 18: 235247

22. Karanth SS, Springall IDR, Lucas S, Levy D, Ashby P, Levene MM, Polak JM (1989) Changes in nerves and neuropeptides in 
skin from 100 leprosy patients investigated by immunocytochemistry. J Pathol 156: 15-26

23. Abraham RR, Abraham RM, Wynn V (1986) Autonomic and electrophysiological studies in patients with signs or symptoms of diabetic neuropathy. Electroencephalogr Clin Neurophysiol 63: 223230

24. Levy DM, Abraham RR, Abraham RM (1987) Small- and largefiber involvement in early diabetic neuropathy: a study with the medial plantar response and sensory thresholds. Diabetes Care 10: $441-447$

25. Ewing DJ, Clarke BF (1982) Diagnosis and management of diabetic autonomic neuropathy. Br Med J 285: 916-918

26. Faerman I, Faccio F, Calb I, Razumny J, Franco N, Dominguez A, Podestá HA (1982) Autonomic ncuropathy in the skin: a histological study of the sympathetic nerve fibres in diabetic anhidrosis. Diabetologia 22: $96-99$

27. Johnson PC, Doll SC (1984) Dermal nerves in human diabetic subjects. Diabetes $33: 244.250$

28. Waxman SG, Brill MH, Geschwind N, Sabin TD, Lettvin JY (1976) Probability of conduction deficit as related to fiber length in random-distribution models of peripheral neuropathics. J Neurol Sci 29: $39-53$

29. Morley GK, Mooradian AD, Levine AS, Morley JE (1984) Mechanism of pain in diabetic peripheral neuropathy: effect of glucose on pain perception in humans. Am J Med 77: 79-82

30. Aronin N, Leeman SE, Clements RS Jr (1987) Diminished flare response in neuropathic diabetic patients: comparison of effects of substance P, histamine, and capsaicin. Diabetes 36: 11391143

31. Parkhouse N, Le Quesne PM (1988) Quantitative objective assessment of peripheral nociceptive $C$ fibre function. J Neurol Neurosurg Psychiatry 51: 28-34
32. Jakobsen J, Sidenius P, Bracndgaard H (1986) A proposal for a classification of neuropathies according to their axonal transport abnormalities. J Neurol Neurosurg Psychiatry 49: 986-990

33. Marini P, Vitadello M, Bianchi R, Triban C, Gorio A (1986) Impaired axonal transport of acetylcholinesterase in the sciatic nerve of alloxan-diabetic rats: effects of ganglioside treatment. Diabetologia 29: 254-258

34. Newrick PG, Wilson AJ, Jakubowski J, Boulton AJM, Ward JD (1986) Sural nerve oxygen tension in diabetes. Br Med J 293: 1053-1054

35. Sima AAF, Bril V, Nathaniel V, McEwen TAJ, Brown MB, Lattimer SA, Greene DA (1988) Regeneration and repair of myelinated fibers in sural-nerve biopsy specimens from patients with diabetic neuropathy treated with sorbinil. N Engl J Med 319: $542-548$

36. Consensus statement: report and recommendations of the San Antonio conference on diabetic neuropathy (1988) Diabetes Care 11: $592-597$

37. Lawrence RD (1965) The diabetic life: a concise practical manual, 17 th edn. Churchill, London, p 157

Received: 2 December 1988

and in revised form: 11 April 1989

Dr. D. M. Levy

Department of Diabetes and Endocrinology

Central Middlesex Hospital

Acton Lane

London NW10 7:NS

UK 